\title{
Multiscale Simulation for Highly Oscillatory Parabolic Model
}

\author{
Meiling Sun* \\ Department of Fundamental Courses \\ Nantong Vocational College \\ Nantong, 226007, China \\ ${ }^{*}$ Corresponding author
}

\author{
Geliang Chen \\ State Grid Nantong Power Supply Company \\ Nantong, 226007, China
}

\begin{abstract}
A multiscale finite element simulation is presented to solve a parabolic problem efficiently. This parabolic model has highly oscillatory coefficient, which make the conventional methods expensive costs or bad behaviors. The new multiscale finite element scheme, whose multiscale basis functions may reflect the local strong oscillation, can acquire good simulation on the macroscopical scale. The Euler backward difference time discretization is applied. Our method just computes on the coarse grids and saves plenty of computer resources, and it obtains convergent and controllable errors with the time iterations.
\end{abstract}

Keywords-multiscale finite element method; parabolic model; singular perturbation; high oscillation; Euler backward difference

\section{INTRODUCTION}

Parabolic problems with high oscillation are widely appeared in fluid mechanics, molecular dynamics, heat transfer and image processing, etc[1]. They have small perturbed parameters, which cause the boundary layers and strong oscillations in the problem.

Their highly-resolved solutions may be computationally expensive or even infeasible due to the great number of fine cells, so conventional methods cannot solve them effectively. As such, some reduction techniques are particularly interested in order to decrease the computational cost, and at the meantime, to acquire the good accuracy.

Hou and $\mathrm{Wu}[2]$ proposed the multiscale finite element method(MsFEM) initially, by solving the local problem to get the multiscale basis functions. After that, the multiscale computations have made many progresses[3-9]. Efendiev, Galvis and Hou[6] presented the generalized multiscale finite element method(GMsFEM) to construct the offline and online space, and extended MsFEM to more general problems. This paper author Sun[8] applied the multiscale basis functions for the singular perturbation on graded meshes, and it was shown to reduce the boundary layer errors remarkably. Jiang, Presho and Huang[9] studied an adapted Petrov-Galerkin MsFEM, which provided the more flexibilities in constructing the function spaces and eliminated the cell resonance effect to improve its convergence and accuracy.

The multiscale parabolic problem is hot and hard spots in recent scientific computation. We consider an initial and boundary value problem(IBVP):

$$
\begin{cases}\frac{\partial u}{\partial t}-\nabla \cdot\left(k_{\varepsilon}(x) \nabla u\right)=f, & \Omega \in(0,1) \times(0,1) \bigcup t \in(0, T] ; \\ u(x ; t)=g, & \partial \Omega \bigcup t \in(0, T] ; \\ u(x ; 0)=u_{0}, & \bar{\Omega} \bigcup t=0,\end{cases}
$$

where $u$ is the solution, $t$ is time, $k_{\varepsilon}(\boldsymbol{x})$ is the highly oscillatory coefficient and is dependent on the scale parameter $\varepsilon(\varepsilon \square 1), \boldsymbol{x}$ is the two-dimensional space variable, $f$ is right-side, $g$ is boundary, $u_{0}$ is initial value, and $\Omega, \partial \Omega$ are the domain and its boundary. This problem is well-posed under some conditions, but its analytical expression is unavailable. So the numerical and accurate solution is our goal in this paper.

\section{STANDARD FEM AND MULTISCALE FEM FOR PARABOLIC PROBLEM}

\section{A. Variational Form and Finite Element Scheme}

The variational form of (1) is to seek $u \in H^{1}(\Omega)$ such that

$$
\left(\frac{\partial u}{\partial t}, v\right)+a(u, v)=(f, v), \quad \forall v \in H^{1}(\Omega), t \in(0, T],
$$

where $a(u, v)=\int_{\Omega} k_{\varepsilon}(x)\left(\frac{\partial u}{\partial x} \frac{\partial v}{\partial x}+\frac{\partial u}{\partial y} \frac{\partial v}{\partial y}\right) \mathrm{d} \boldsymbol{x},(f, v)=\int_{\Omega} f v \mathrm{~d} \boldsymbol{x}$, and $H^{1}$ is the first order differentiable and square-integrable space. The corresponding matrix form is $\boldsymbol{M} \frac{\mathrm{d} u}{\mathrm{~d} t}+\boldsymbol{A} u=\boldsymbol{F}$, where its element $\quad M_{i j}=\int_{\Omega} \varphi_{i} \varphi_{j} \mathrm{~d} \boldsymbol{x}$, $A_{i j}=\int_{\Omega} k_{\varepsilon}(x) \nabla \varphi_{i} \cdot \nabla \varphi_{j} \mathrm{~d} x, F_{i}=\int_{\Omega} f \varphi_{i} \mathrm{~d} \boldsymbol{x}$, and $\varphi_{i}$ is the standard finite element basis function. 
We divide the domain $\Omega$ into several sub-domain $\Omega_{k}$ with $\bigcup \Omega_{k}=\Omega$, and let $K^{h}$ to be the rectangular uniform grids partition, thus in each element $\left[x_{i}, x_{i+1}\right] \times\left[y_{j}, y_{j+1}\right]$ is the corresponding nodal coordinates and four standard basis functions are $\varphi_{1}=(1-\xi)(1-\eta), \varphi_{2}=\xi(1-\eta), \varphi_{3}=\xi \eta$, $\varphi_{4}=(1-\xi) \eta$, where the variable $\xi=\left(x-x_{i}\right) / h_{x}$, $\eta=\left(y-y_{j}\right) / h_{y}$, mesh size $h_{x}=x_{i+1}-x_{i}$, $h_{y}=y_{j+1}-y_{j}$, and $\mathrm{d} x=h_{x} \mathrm{~d} \xi, \mathrm{d} y=h_{y} \mathrm{~d} \eta$. In this way, the local mass matrix element

$$
\begin{gathered}
M_{11}=\int_{x_{i}}^{x_{i+1}} \int_{y_{j}}^{y_{j+1}} \varphi_{1} \varphi_{1} \mathrm{~d} x \mathrm{~d} y \\
=\int_{0}^{1} \int_{0}^{1}(1-\xi)^{2}(1-\eta)^{2} h_{x} h_{y} \mathrm{~d} \xi \mathrm{d} \eta=\frac{1}{9} h_{x} h_{y},
\end{gathered}
$$

and $M_{12}, \ldots, M_{44}$ are obtained similarly. The local stiffness matrix element

$$
\begin{gathered}
A_{11}=\int_{x_{i}}^{x_{i+1}} \int_{y_{j}}^{y_{j+1}} k_{\varepsilon}(\boldsymbol{x})\left(\frac{\partial \varphi_{1}}{\partial x} \frac{\partial \varphi_{1}}{\partial x}+\frac{\partial \varphi_{1}}{\partial y} \frac{\partial \varphi_{1}}{\partial y}\right) \mathrm{d} x \mathrm{~d} y \\
=\int_{0}^{1} \int_{0}^{1}\left(k_{1} \varphi_{1}+\cdots+k_{4} \varphi_{4}\right)\left[(1-\eta)^{2} \frac{h_{y}}{h_{x}}+(1-\xi)^{2} \frac{h_{x}}{h_{y}}\right] \mathrm{d} \xi \mathrm{d} \eta \\
=\frac{k_{1}}{8}\left(\frac{h_{y}}{h_{x}}+\frac{h_{x}}{h_{y}}\right)+\frac{k_{2}}{8}\left(\frac{h_{y}}{h_{x}}+\frac{h_{x}}{3 h_{y}}\right)+\frac{k_{3}}{24}\left(\frac{h_{y}}{h_{x}}+\frac{h_{x}}{h_{y}}\right)+\frac{k_{4}}{8}\left(\frac{h_{y}}{3 h_{x}}+\frac{h_{x}}{h_{y}}\right),
\end{gathered}
$$
and $A_{12}, \ldots, A_{44}$ are similar. The local force vector element

$$
\begin{gathered}
F_{1}=\int_{x_{i}}^{x_{i+1}} \int_{y_{j}}^{y_{j+1}} f \varphi_{1} \mathrm{~d} x \mathrm{~d} y=\int_{0}^{1} \int_{0}^{1}\left(f_{1} \varphi_{1}+\cdots+f_{4} \varphi_{4}\right) \varphi_{1} h_{x} h_{y} \mathrm{~d} \xi \mathrm{d} \eta \\
=\left(\frac{1}{9} f_{1}+\frac{1}{18} f_{2}+\frac{1}{36} f_{3}+\frac{1}{18} f_{4}\right) h_{x} h_{y}, \text { and } F_{2}, F_{3}, F_{4}
\end{gathered}
$$
are similar. In turn, we solve the global matrix system to acquire the Galerkin finite element solution $u_{g}$.

\section{B. Multiscale Finite Element Scheme}

Now we present the new multiscale FEM to settle the parabolic model. The corresponding variational form is to seek $u_{h} \in U^{h}$ such that

$$
\left(\frac{\partial u_{h}}{\partial t}, v\right)+a\left(u_{h}, v\right)=(f, v), \quad \forall v \in U^{h}, t \in(0, T]
$$

where $u_{h}$ is the MsFEM solution, and the multiscale space $U^{h}=\operatorname{span}\left\{\phi_{i}, \forall K \in K^{h}\right\}$ is built by the multiscale basis functions $\phi_{i}$.
Being different from the standard bases $\varphi_{i}$, the multiscale bases $\phi_{i}$ are not expressed analytically. They are obtained from the sub-problem on coarse element $K$

$$
\left\{\begin{array}{cl}
-\nabla \cdot\left(k_{\varepsilon}(x) \nabla \phi_{i}\right)=0, & \text { in } K \\
\phi_{i}(x)=l_{i}, & \text { on } \partial K
\end{array}\right.
$$

Set the local boundary condition, i.e., $\phi_{i}\left(x_{j}, y_{j}\right)=1$ when $i=j, \phi_{i}\left(x_{j}, y_{j}\right)=0$ when $i \neq j$, and $\phi_{i}\left(x_{j}, y_{j}\right)$ is varying linearly on $\partial K$. Since the sub-problem (4) has the same differential operator as the original problem (1), thus the multiscale basis functions $\phi_{i}$ can show the local information such as the oscillations, which is not available from the former standard bases $\varphi_{i}$.

\section{Backward Difference Time Discretization}

Now we make the time discretization. We know that the Euler forward difference does not work, so here we outline the Euler backward difference for the model. Note that this backward difference is an inexplicit scheme.

$$
\begin{aligned}
& \boldsymbol{M} \frac{u^{(k+1)}-u^{(k)}}{\Delta t}+\boldsymbol{A} u^{(k+1)}=\boldsymbol{F} \\
\Rightarrow & \left(u^{(k+1)}-u^{(k)}\right)+\Delta t \boldsymbol{M}^{-1} \boldsymbol{A} u^{(k+1)}=\Delta t \boldsymbol{M}^{-1} \boldsymbol{F} \\
\Rightarrow & u^{(k+1)}=\left(\boldsymbol{I}+\Delta t \boldsymbol{M}^{-1} \boldsymbol{A}\right)^{-1} \cdot u^{(k)}+\left(\boldsymbol{I}+\Delta t \boldsymbol{M}^{-1} \boldsymbol{A}\right)^{-1} \cdot \Delta t \boldsymbol{M}^{-1} \boldsymbol{F} \\
= & (\boldsymbol{M}+\Delta t \boldsymbol{A})^{-1} \boldsymbol{M} \cdot u^{(k)}+\Delta t(\boldsymbol{M}+\Delta t \boldsymbol{A})^{-1} \boldsymbol{F}
\end{aligned}
$$

$$
\text { Set } \boldsymbol{S}=(\boldsymbol{M}+\Delta t \boldsymbol{A})^{-1} \boldsymbol{M}, \boldsymbol{G}=\Delta t(\boldsymbol{M}+\Delta t \boldsymbol{A})^{-1} \boldsymbol{F},
$$
then

$$
u^{(k+1)}=\boldsymbol{S} \cdot \boldsymbol{u}^{(k)}+\boldsymbol{G}
$$

Thus in this iterative way we can acquire the multiscale parabolic solution $u_{h}^{(k+1)}$ along with the time eclipsing.

\section{NUMERICAL EXPERIMENT}

In this section, we use the standard FEM and the multiscale FEM to solve the parabolic model (1), respectively. The codes are compiled by Matlab2012. We set the domain $\Omega \in(0,1) \times(0,1)$, and the coarse element partition number $N_{x}=N_{y}=8$, in each coarse element the fine partition number $n_{x}=n_{y}=8$. Note that the standard FEM computes on the fine grids $\left(N_{x} \times n_{x}+1\right) \times\left(N_{y} \times n_{y}+1\right)=N_{\text {globenode, }}$, while the multiscale FEM just computes on the coarse grids $\left(N_{x}+1\right) \times\left(N_{y}+1\right)=N_{\text {coarsenode. }}$ The latter method is shown to provide good accuracy, at the same time it saves the required computer memory and time. 
We take the very small parameter $\varepsilon$ in the benchmark model from Stanford university permeableness coefficient $k_{\varepsilon}(x)$, see Figure I. And the right side $f=1$, boundary $g=0$, initial value $u_{0}=\sin \pi x \cdot \sin \pi y$.
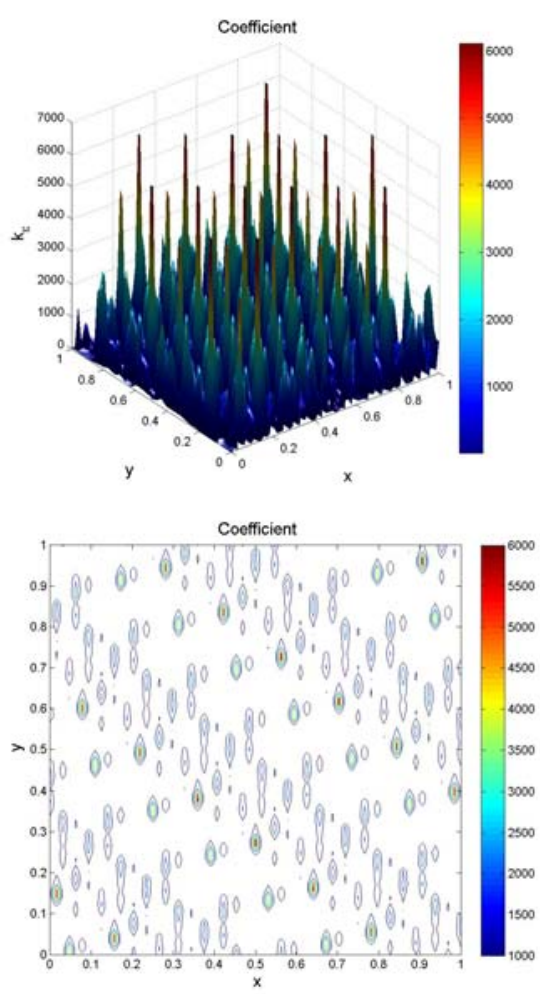

FIGURE I. THE HIGHLY OSCILLATORY COEFFICIENT AND ITS CONTOUR

The absolute error and relative error of $L^{2}$ norm are as followed

$$
\begin{gathered}
\left\|u-u_{h}\right\|_{L^{2}}=\left(\int_{\Omega}\left(u-u_{h}\right)^{2} \mathrm{~d} x \mathrm{~d} y\right)^{1 / 2}, \\
\frac{\left\|u-u_{h}\right\|_{L^{2}}}{\|u\|_{L^{2}}}=\frac{\left(\int_{\Omega}\left(u-u_{h}\right)^{2} \mathrm{~d} x \mathrm{~d} y\right)^{1 / 2}}{\left(\int_{\Omega} u^{2} \mathrm{~d} x \mathrm{~d} y\right)^{1 / 2}}
\end{gathered}
$$

Since we don't have exact solution for the model (1), we take the FEM solution on very fine grid as the "exact" solution, and $u_{h}$ is the multiscale solution.

Set the time step $\Delta t=0.001$, and iteration number is 30 . After iterations, the standard FEM solution $u_{g}^{(30)}$ and the multiscale FEM solution $u_{h}^{(30)}$ are very similar, see Figure II. While we should keep in mind that the latter multiscale method is obtained from the coarse grid computations, and it just costs small amount of computer resource and time to acquire the good simulation.
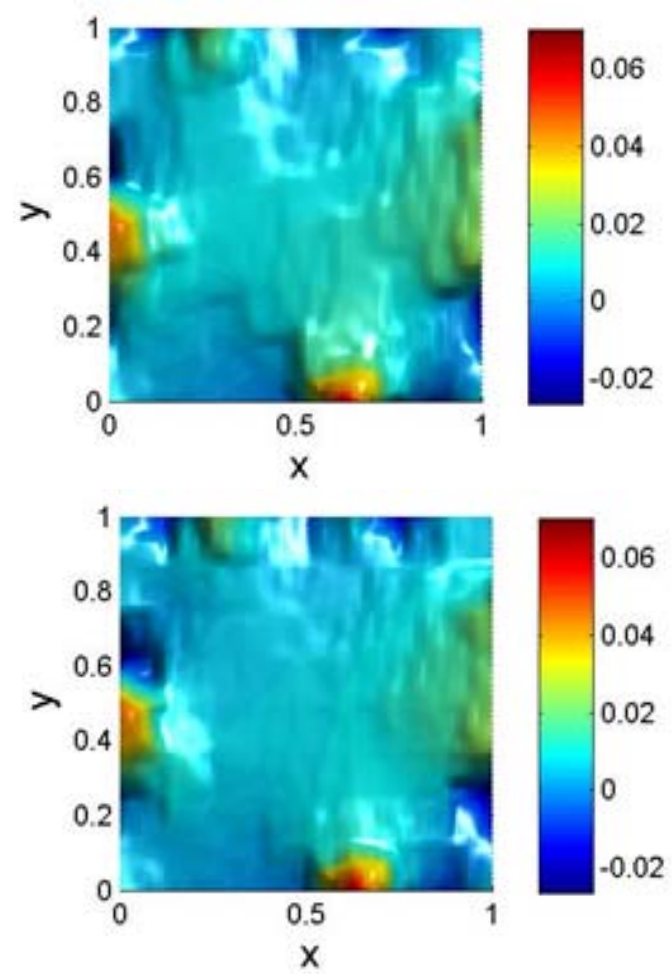

FIGURE II. STANDARD FEM SOLUTION $u_{g}^{(30)}$ AND MSEFM SOLUTION $u_{h}^{(30)}$, RESPECTIVELY
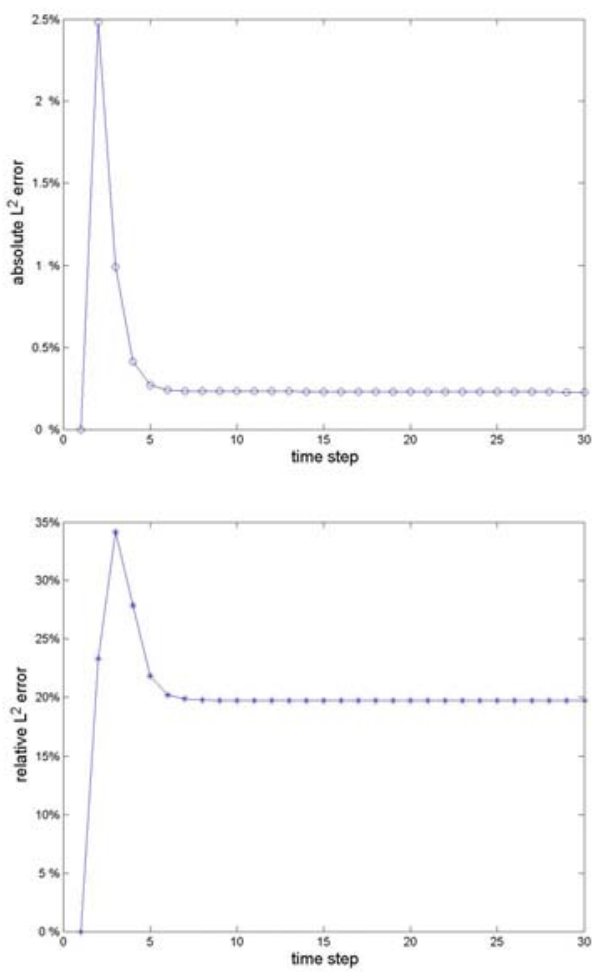

FIGURE III. ABSOLUTE ERROR AND RELATIVE ERROR OF L2 NORM BY MSFEM 
From Figure III we provide the absolute error and relative error of $L^{2}$ norm by our MsFEM with the time eclipsing, and this means the numerical solution behaves well to present the stable and convergent approximations.

\section{CONCLUSIONS}

In this paper, we present a multiscale simulation to solve a parabolic model with highly oscillatory coefficient. The multiscale finite element scheme and Euler backward time discretization are applied. With the help of the multiscale basis functions, we save computer costs to get good and convergent simulations for the parabolic problem.

\section{ACKNOWLEDGMENT}

Research is supported by National Natural Science Foundation of China(Grant No.11301462), University Science Research Project of Jiangsu Province(Grant No.13KJB110030).

\section{REFERENCES}

[1] J. J. Miller, E. O’Riordan, and G. I. Shishkin, Fitted numerical methods for singular perturbation problems[M], World Scientific, Singapore, 1996.

[2] T. Y. Hou and X. H. WU, A multiscale finite element method for elliptic problems in composite materials and porous media[J], J Comput Phys, 1997, 134(1): 169-189.

[3] X. Cai, D. L. CAI, R. Q. Wu, et al, High accuracy non-equidistant method for singular perturbation reaction-diffusion problem[J], Appl Math Mech, 2009, 30(2): 175-182.

[4] X. Y. Yue and W. N. E, The local microscale problem in the multiscale modeling of strongly heterogeneous media: effect of boundary conditions and cell size[J], J Comput Phys, 2007, 222(2): 556-572.

[5] S. Jiang and Y. Q. Huang, Numerical investigation on the boundary conditions for the multiscale base functions[J], Commun Comput Phys, 2009, 5(5): 928-941.

[6] Y. Efendiev, J. Galvis and T. Y. Hou, Generalized multiscale finite element methods(GMsFEM)[J], J Comput Phys, 2013, 251: 116-135.

[7] W. B. Deng and H. J. Wu, A combined finite element and multiscale finite element method for the multiscale elliptic problems[J], Multiscale Model Simul, 2014, 12(4): 1424-1457.

[8] M. L. Sun and S. Jiang, Multiscale basis functions for singular perturbation on adaptively graded meshes[J], Adv Appl Math Mech, 2014, 6(5): 604-614.

[9] S. Jiang, M. Presho and Y. Q. Huang, An adapted Petrov-Galerkin multiscale finite element for singularly perturbed reaction-diffusion problems[J], Int $\mathrm{J}$ Comput Math, 2015, 12 pages, DOI: 10.1080/00207160.2015.1041935 